\title{
Simulations and Experiments on the Effective Optical Gain of FEC in a GPON Uplink
}

\author{
Yanchun Yi, Stefaan Verschuere, Zhe Lou, Peter Ossieur, Johan Bauwelinck, Xing-Zhi Qiu, and Jan Vandewege
}

\begin{abstract}
This letter studies the effective optical gain of Reed-Solomon (RS) forward-error correction (FEC) in a burst-mode gigabit passive optical network (GPON) uplink. Numerical simulations are made of the performance of an RS (255, 239) FEC code. For the first time, FEC performance is measured in a 1.25-Gb/s burst-mode GPON uplink in the presence of mode partition noise (MPN). Measurements show that the effective optical gain of $\mathrm{RS}(255,239)$ can be considerably higher than $2.7 \mathrm{~dB}$ when MPN dominates.
\end{abstract}

Index Terms-Burst-mode, effective optical gain, Fabry-Pérot (FP) laser, forward-error correction (FEC), gigabit passive optical network (GPON), mode partition noise (MPN).

\section{INTRODUCTION}

$\mathbf{T}$ HE passive optical network (PON) technology is regarded as the most cost-efficient solution to the last-mile problem. A symmetric $1.25-\mathrm{Gb} / \mathrm{s}$ gigabit PON (GPON) system was validated by integrating burst-mode physical media dependent chips developed in [1]-[3]. This effort yielded the first public GPON demonstration and showed a very promising performance [4]. To further minimize the cost per subscriber, a Fabry-Perot (FP) laser is preferred for the optical network termination (ONT). As the multimode spectrum of the FP laser causes mode partition noise (MPN), which can be the limiting factor instead of the optical power budget in a dispersive GPON uplink with a data rate of $1.25 \mathrm{~Gb} / \mathrm{s}$, the ITU-T Recommendation G.984.2 [5] proposes to use forward-error correction (FEC) to reduce the associated penalty. The effective optical gain, which is defined as the difference of optical power at the receiver input, with and without FEC, for a bit-error ratio (BER) $=10^{-10}$, achieved with FEC allows for a longer physical reach or a higher split ratio. Moreover, people are also considering the use of FEC to eliminate the need for an avalanche photodiode (APD) receiver at the optical line termination (OLT). Using a PIN photodiode receiver instead, the required sensitivity of the OLT burst-mode receiver still can be ensured. However, the effective optical gain using FEC in burst-mode transmission was unknown and only an estimation was given in ITU-T Recommendation G.984.3, stating that FEC results in a link budget increase of approximately 3-4 dB [6]. Nevertheless, studies about FEC in PON such as [7] are mainly based on theoretical studies

Manuscript received July 13, 2006; revised October 31, 2006.

Y. Yi, P. Ossieur, J. Bauwelinck, X.-Z. Qiu, and J. Vandewege are with the Department of Information Technology, Ghent University, B-9000 Gent, Belgium (e-mail: xingzhi@intec.UGent.be).

S. Verschuere and Z. Lou are with Research and Innovation, Alcatel Bell N.V., B-2018 Antwerp, Belgium.

Digital Object Identifier 10.1109/LPT.2006.888969

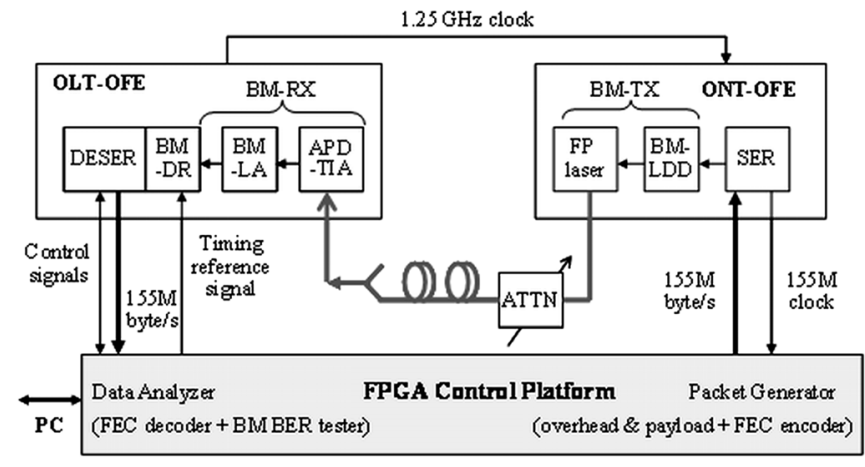

Fig. 1. Experimental setup of an FEC integrated GPON uplink.

of continuous-mode transmission, the burst-mode characteristics of a GPON uplink are not well included. Lacking a close study, people doubt the cost-efficiency of FEC in a GPON uplink though there are already a lot of commercial GPON FEC Codecs available. To really find out the FEC effective optical gain offered by FEC in a GPON uplink, detailed simulations are required which account for the presence of MPN and the influence of burst-mode transmission, as well as experiments on a realistic GPON uplink with integrated FEC. This letter presents numerical simulations of the FEC effective optical gain in a burst-mode GPON uplink, made on a MATLAB/Simulink platform, and experiments of integrating FEC in a $1.25-\mathrm{Gb} / \mathrm{s}$ GPON uplink.

\section{FEC INTEGRATED GPON UPLINK}

Fig. 1 illustrates the experimental setup of the GPON $1.25-\mathrm{Gb} / \mathrm{s}$ burst-mode uplink with integrated FEC. By our knowledge, this is the first public GPON uplink demo setup including FEC. The ONT optical front-end (OFE) contains one burst-mode transmitter (BM-TX), which contains a low-cost FP laser diode and a burst-mode laser diode driver (BM-LDD) [1], following an 8:1 serializer (SER) [8], and the OLT-OFE contains a burst-mode receiver (BM-RX), which consists of a high sensitivity burst-mode APD-transimpedance amplifier (TIA) and a wide dynamic range burst-mode limiting amplifier (BM-LA) designed for instantaneous packet amplitude recovery [2], followed by a burst-mode data recovery (BM-DR) chip. The BM-DR IC contains the high-speed blocks of the line termination and includes the deserializer (DESER) function. It performs the upstream retiming, i.e., the clock phase alignment (CPA) and the burst alignment via delimiter detection. After FEC encoding, the 155-MB/s parallel data outputs from an FPGA-based packet generator are serialized to generate a $1.25-\mathrm{Gb} / \mathrm{s}$ burst-mode data stream. Different FEC schemes for 


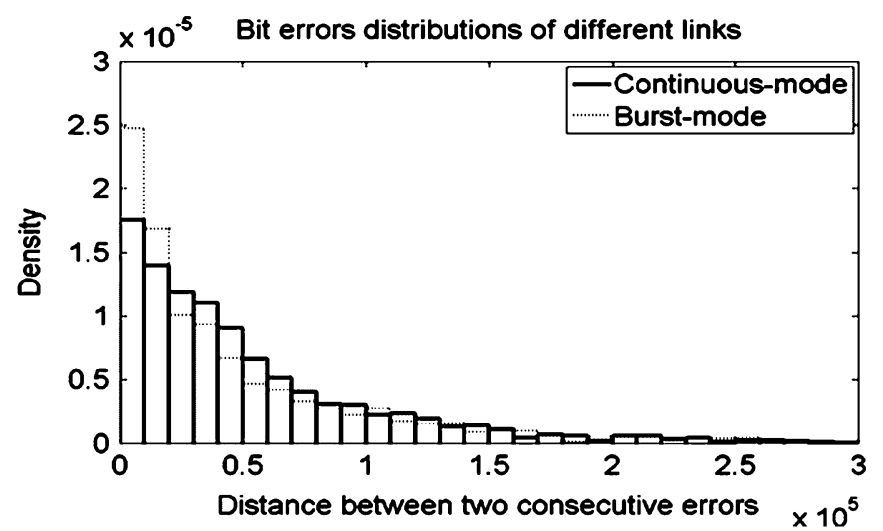

Fig. 2. Bit-error distributions in different links when BER $=1.4 \times 10^{-5}$, the burst-mode receiver acquests the amplitude of the incoming burst from the 12 bits consecutive " 1 " and " 0 " at the beginning with peak detectors.

GPON uplink were compared in the literature such as [7] and the Reed-Solomon (RS) $(255,239)$ FEC code is proposed in G.984.3 [6]. Simulations in this letter also use RS $(255,239)$ FEC to compare with experimental results of the FEC IP core available. After an FEC decoder, an FPGA-based data analyzer measures the burst-mode BER of the recovered incoming packets.

\section{Simulations}

The effective optical gain of the FEC depends on two factors: the bit-error distribution in the link and the slope of the BER curve. In a GPON uplink, as shown in Fig. 1, the BM-LA instantaneously recovers the amplitude of each burst by extracting the decision threshold from a number of preamble bits [2]. Since the length of the preamble is maximum 44 bits at $1.25 \mathrm{~Gb} / \mathrm{s}$ as specified in G.984.2 [5], even when the amplitude of the incoming bursts remains the same, the extracted decision threshold must not be regarded as a constant as in a traditional continuous-mode receiver, but can be approximated as a Gaussian distributed variable according to numerical simulation results. However, the equivalent bandwidth of the noise on the decision threshold is much smaller than the data rate, because the threshold of a complete burst is mainly determined at the start of the burst. Here we assume that the noise on the threshold is correlated over the length of one codeword of the RS $(255,239)$ FEC code. Consequently, one finds a bit-error distribution as shown in Fig. 2 after numerical simulations. The bars with solid lines represent the probability density of the distance between two consecutive errors in a continuous-mode link, and the bars with dashed lines show the probability density of this distance in a burst-mode link. Fig. 2 assumes links with a BER of $1.4 \times 10^{-5}$, which is the range proposed for the RS $(255,239)$ FEC code. It learns that, at this BER, the probability that two consecutive errors have a very short distance between each other is higher in a burst-mode link than in a continuous-mode link. A higher probability to have closely spaced bit errors means that a burst-mode link is more likely to have burst errors. The cause of this phenomenon is that, in a burst-mode receiver, the extracted threshold can deviate from the optimal value from burst to burst. More errors occur in bursts with a less optimum threshold and less in bursts with a more accurate threshold. Since the RS $(255,235)$ FEC
$R S(255,239)$ in different links

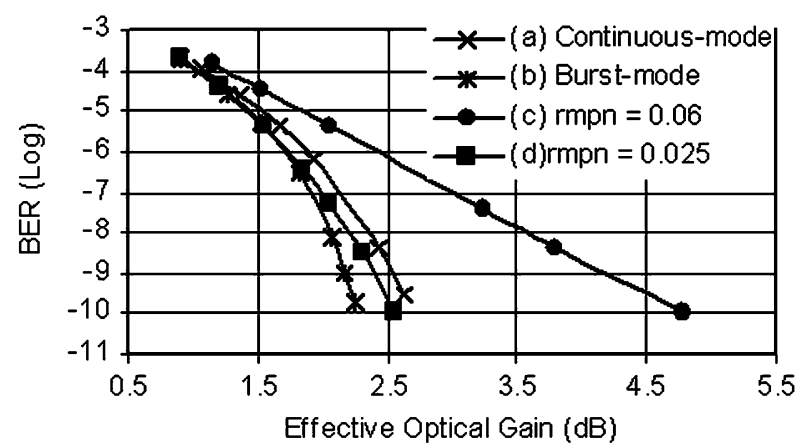

Fig. 3. Effectiveoptical gain of RS $(255,239)$ FEC code as function of BER in different links.

code cannot correct burst errors longer than eight symbols, its effective optical gain in a burst-mode link will be smaller than in a continuous-mode link. This conclusion is proven by the simulation results given in Fig. 3. Curve (a) in Fig. 3 shows the relationship between the effective optical gain of an RS (255, 239) FEC code and the BER in a continuous-mode link. Curve (b) in Fig. 3 shows the effective optical gain of this FEC in a burst-mode link. The simulation shows that the effective optical gain of $\mathrm{RS}(255,239)$ FEC is about $2.7 \mathrm{~dB}$ in a continuous-mode link and about $2.3 \mathrm{~dB}$ in a burst-mode link at a BER of $10^{-10}$. While the burst-mode nature changes the bit-error distribution in a GPON uplink, the MPN changes the slope of the BER curve. According to the accepted MPN descriptions in [9], the MPN can be characterized as additional receiver noise with a properly defined variance $r_{\mathrm{mpn}}$, which is determined by [9]

$$
r_{\mathrm{mpn}}=\frac{k}{\sqrt{2}}\left[1-e^{-\left(\pi B D L \sigma_{\lambda}\right)^{2}}\right]
$$

where $k$ is the mode-partition coefficient, $B$ is the data rate, $D$ is the fiber dispersion, $L$ is the length of fiber, and $\sigma_{\lambda}$ is the root-mean-square spectral width of the FP laser. The MPN penalty $P_{\mathrm{mpn}}$ can be estimated by [9]

$$
P_{\mathrm{mpn}}=-5 \log \left(1-Q^{2} r_{\mathrm{mpn}}^{2}\right)
$$

where $Q$ is the effective signal-to-noise ratio and the BER can be evaluated by

$$
\mathrm{BER}=\frac{1}{2} \operatorname{erfc}\left(\frac{Q}{\sqrt{2}}\right) .
$$

This means that $P_{\mathrm{mpn}}$ increases with BER, in other words, MPN will make the BER curve more flat. The larger $r_{\mathrm{mpn}}$, the flatter the BER curve becomes. Hence, there will be room for effective FEC optical gain in a GPON uplink with MPN. The simulated curve (d) in Fig. 3 shows an FEC effective optical gain around $2.5 \mathrm{~dB}$ at BER $=10^{-10}$ in a GPON uplink when $r_{\text {mpn }}=0.025$. At higher BER, curves (b) and (d) are very close since the corresponding $Q$ and $P_{\text {mpn }}$ are small. At lower BER, curve (d) in Fig. 3 shows a larger effective optical gain than curve (b) as the $P_{\text {mpn }}$ increases with decreasing BER. When $r_{\text {mpn }}=0.06$, the effective optical gain is about $4.8 \mathrm{~dB}$ according to the simulated curve (c) in Fig. 3. As the MPN is uncorrelated over 3 ns (about 4 bits when the data rate is $1.25 \mathrm{~Gb} / \mathrm{s})$, the Gaussian noise is 


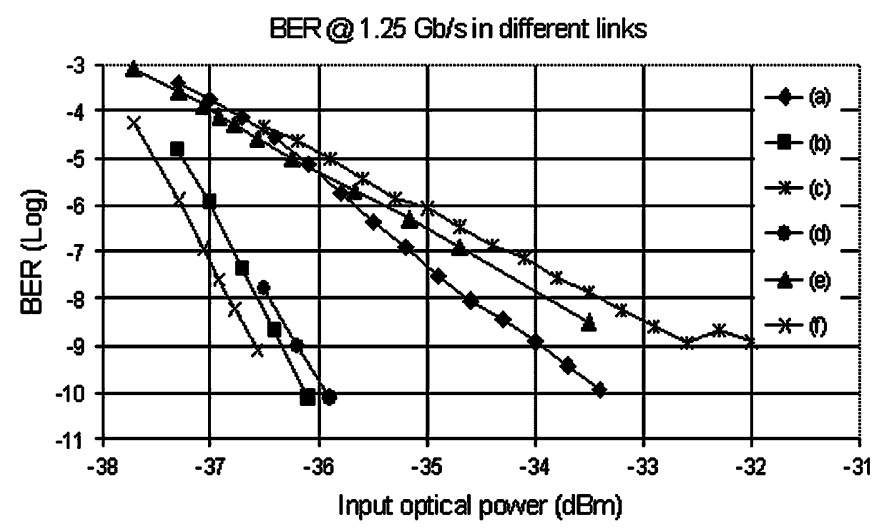

Fig. 4. Effective optical gain of RS $(255,239)$ FEC code as function of BER in different links.

also a reasonable approximation of the MPN when RS (255, 239) FEC code can correct burst errors up to eight symbols.

\section{EXPERIMENT RESULTS}

An FP laser with a central wavelength around $1307 \mathrm{~nm}$ was used for experiments to investigate the effective optical gain of RS $(255,239)$ FEC code. The effective optical gain of the system employing FEC is defined as the difference of optical power at the receiver input, with and without FEC, for a BER = $1 \times 10^{-10}$. No notable MPN penalty is observed even with $20-\mathrm{km}$ G.652 fiber, when the central wavelengths of the FP lasers used are within the zero dispersion window of the fiber (1302-1322 nm). However, ITU-T G.984.2 specifies the ONT transmitter operating wavelength in the range of $1260-1360 \mathrm{~nm}$, so in the worst case, the MPN penalty cannot be negligible. To emulate such a condition with the given 1307-nm FP laser, $3.1 \mathrm{~km}$ of G.653 dispersion-shifted fiber (DSF) with dispersion of $-20 \mathrm{ps} / \mathrm{nm} \cdot \mathrm{km}$ at $1307 \mathrm{~nm}$ was inserted into the uplink. This is equivalent to the dispersion of a 1360-nm FP laser over 12-km G.652 fiber with a dispersion of $5 \mathrm{ps} / \mathrm{nm} \cdot \mathrm{km}$, which can happen in a G.984.2 compliant system. Fig. 4 plots the measured BM-BER (at APD gain $=6$ ) of the GPON uplink with 3.1-km DSF fiber. Curve (a) is the BER curve of the FP laser plus 20-km G.652 fiber without FEC. Curve (b) Fig. 4 is the BER curve of FP laser plus 20-km G.652 with FEC, curve (c) is the BER curve of the FP laser plus 3.1-km G.653 DSF without FEC, and curve (d) is the BER curve of FP laser plus 3.1-km DSF with FEC. This illustrates the penalty caused by MPN, and also the error correction capability of the RS $(255,239)$ FEC code. For comparison, simulation results of $r_{\mathrm{mpn}}=0.038$ are plotted as curves (e) (without FEC) and (f) (with FEC).

According to theoretical analysis, the RS $(255,239)$ code can improve the BER from $10^{-4}$ to $10^{-12}$ in a continuous-mode digital optical communication system when the bit errors are discrete. In our experiments, only $10^{-5}$ to $10^{-11}$ improvement has been observed, which means that there are more burst errors in a burst-mode GPON uplink. It agrees with simulation results given in Figs. 2 and 3. We also learn from Fig. 4 that BER curve (c) is flatter than curve (a) and shows a BER floor over $10^{-9}$ eventually. This result confirms the existence of the MPN. It proves the simulation results that the RS $(255,239)$ FEC code
TABLE I

EFFECTIVE OPTICAL GAIN IN DIFFENT LINKS

\begin{tabular}{|c|c|c|}
\hline Fiber $(\mathbf{k m})$ & FEC gain & RX sensitivity $(\mathbf{d B m})$ \\
\hline $20(\mathrm{G} .652)$ & no FEC & $-33.4(\mathrm{FP})$ \\
\hline $20(\mathrm{G} .652)$ & $\mathrm{G}=2.7 \mathrm{~dB}$ & $-36.1(\mathrm{FP}+\mathrm{FEC})$ \\
\hline $3.1(\mathrm{G} .653 \mathrm{DSF})$ & no FEC & BER floor \\
\hline $3.1(\mathrm{G} .653 \mathrm{DSF})$ & $\mathrm{G}>3.6 \mathrm{~dB}$ & -36 \\
\hline
\end{tabular}

will have more effective optical gain in an MPN limited link. Table 1 summarizes the measured effective optical gain of an RS $(255,239)$ FEC code on the GPON uplink.

\section{CONCLUSION}

According to simulation and experimental results, the effective optical gain of the RS $(255,239)$ FEC code on the GPON uplink is about $2.3-2.7 \mathrm{~dB}$, as the burst-mode transmission will reduce the FEC gain. However, the effective optical gain can vary when different receivers are used. In an MPN-limited link, this gain is more significant since the MPN penalty is larger at lower BER. This allows us to extend the PON reach despite using cheap FP lasers, or to nearly double the number of subscribers in a given PON topology. FP lasers in combination with RS $(255,239)$ FEC code can be used as a cost saving optical source for the GPON uplink, and guarantee a high sensitivity, which is specified in the ITU-T G.984.2 recommendation [5], with extended physical reach.

\section{ACKNOWLEDGMENT}

The authors would like to thank Telecom Italia for providing the RS decoder IP and H. Krimmel of Alcatel R\&I Stuttgart for supporting the FEC experiment.

\section{REFERENCES}

[1] J. Bauwelinck, W. Chen, D. Verhulst, Y. Martens, P. Ossieur, X.-Z. Qiu, and J. Vandewege, "A high-resolution burst-mode laser transmitter with fast and accurate level monitoring for $1.25 \mathrm{~Gb} / \mathrm{s}$ upstream GPONs," IEEE J. Solid-State Circuits, vol. 40, no. 6, pp. 1322-1330, Jun. 2005.

[2] P. Ossieur, D. Verhulst, Y. Martens, W. Chen, J. Bauwelinck, X.-Z. Qiu, and J. Vandewege, "A 1.25-Gb/s burst-mode receiver for GPON applications," IEEE J. Solid-State Circuits, vol. 40, no. 5, pp. 1180-1189, May 2005.

[3] D. Verhulst, X. Yin, J. Bauwelinck, P. Ossieur, X. Z. Qiu, and J. Vandewege, "A robust phase detector for 1.25 Gbps burst-mode data recovery," IEICE Electron. Express, vol. 1, no. 18, pp. 562-567, Dec. 2004.

[4] X. Z. Qiu, P. Ossieur, J. Bauwelinck, Y. Yi, D. Verhulst, J. Vandewege, B. De Vos, and P. Solina, "Development of GPON upstream physical media dependent prototypes," J. Lightw. Technol., vol. 22, no. 11, pp. 2498-2508, Nov. 2004.

[5] Gigabit-Capable Passive Optical Networks (GPON): Physical Media Dependent (PMD) Layer Specification, ITU-T Recommendation G.984.2, 2003.

[6] Gigabit-Capable Passive Optical Networks (G-PON): Transmission Convergence Layer Specification, ITU-T Recommendation G.984.3, 2003.

[7] X. Liu, C. Lu, and T. H. Cheng, "Forward error control in passive optical network," in Tech. Dig. Optical Fiber Communication Conf. (OFC/NFOEC), Mar. 6-11, 2005, vol. 1.

[8] D. Verhulst et al., "Upstream experiments on the gigabit PON physical medium layer," in Tech. Dig. Fiber Communication Conf. (OFC/ NFOEC), Mar. 6-11, 2005, Paper OFI2.

[9] G. P. Agrawal, P. J. Anthony, and T. M. Shen, "Dispersion penalty for $1.3 \mu \mathrm{m}$ lightwave systems with multimoded semiconductor lasers," $J$. Lightw. Technol., vol. 6, no. 5, pp. 620-625, May 1988. 